\title{
Ultrafast Structural Dynamics in Condensed Matter
}

\author{
Paul Beauda*, Steven L. Johnson ${ }^{\mathrm{a}}$, Ekaterina Vorobevaa, Christopher J. Milne ${ }^{\mathrm{b}}$, Andrin Caviezela, \\ Simon O. Mariager ${ }^{a}$, Raquel A. De Souza ${ }^{a}$, Urs Stauba, and Gerhard Ingold ${ }^{a}$
}

\begin{abstract}
We review our recent activity in the field of photo-induced structural dynamics in crystalline solids studied using femtosecond X-ray diffraction techniques.
\end{abstract}

Keywords: Coherent phonons · Femtosecond X-rays · Phase transitions · Strongly correlated electron systems - Ultrafast structural dynamics

\section{Introduction}

Very short, intense pulses of light have the ability to excite matter far from equilibrium on extremely short time scales. The ensuing real-time dynamics have the potential to reveal unique insights into the complex interplay of forces that ultimately determine the properties of materials. To obtain both the spatial and temporal resolution to resolve the dynamics of long-range order on the atomic scale, femtosecond laserpump/X-ray-probe methods have proven to be a highly versatile tool. They can capture the dynamics of electron, spin and structure directly in the time domain. This allows investigations of correlations in real space as they develop in time. ${ }^{[1-7]}$

FEMTO is a tunable undulator source for femtosecond X-rays in the range 5-12 $\mathrm{keV}$ in operation at the microXAS beamline at the Swiss Light Source (SLS). ${ }^{[8]}$ Two classes of experiments are performed at FEMTO: time-resolved X-ray diffraction (trXRD) in the solid state, and X-ray absorption (trXAS) experiments in photochemistry. In crystalline systems trXRD provides information about coherent transient changes of long-range order, whereas
XAS is a complementary technique that measures local electronic and structural changes. In both cases a typical measurement allows the reconstruction of atomic distances with a precision of $<0.01 \AA$. The element specificity of trXAS has been applied to study electronic and structural dynamics during the fs magnetization of metal-ligand complexes, where an electron in a low-spin ground-state is optically excited and eventually joins with another metal-centered electron to generate a paramagnetic high-spin excited state. ${ }^{[9,10]}$

The in-house research of the FEMTO team concentrates on the ultrafast structural dynamics of laser-excited solids. In a crystalline solid the local structural changes of dilute laser-excited impurities have been studied by time-resolved X-ray absorption near-edge structure (XANES). ${ }^{[1]}$ To study the dynamics of long-range order, initial experiments focused on the development of suitable trXRD techniques and on understanding fundamental aspects of electron-phonon interactions in model systems where the unit cell contains only two or three atoms. In collaboration with the SLSRESOXS group (resonant soft X-ray spectroscopy) we have recently expanded our research to include structural dynamics of strongly correlated electron systems (SC$\mathrm{ES})$ in the vicinity of phase transitions. ${ }^{[12]}$ In this report we will give an overview of the source and the methods involved, and discuss our activities in this rapidly growing area of research.

\section{Femtosecond X-rays at the SLS}

${ }^{*}$ Correspondence: Dr. P. Beaud ${ }^{\mathrm{a}}$

Tel.: + 41563104121

Fax: + 41563103151

E-mail: paul.beaud@psi.ch

aSwiss Light Source

Paul Scherrer Institut

$\mathrm{CH}-5232$ Villigen

bLaboratoire de Spectroscopie Ultrarapide

Ecole Polytechnique Fédérale de Lausanne

$\mathrm{CH}-1015$ Lausanne Pred frequency, polariz. The tion, coherence and time structure of this radiation can be precisely tailored by controlling the electron dynamics in phase space. There are several ways to gener- ate ultrashort X-rays at undulator sources for instance by 'slicing' or compressing the electron pulses. These approaches all rely on a dispersive element to exploit an energy-momentum correlation within the bandwidth of the electron pulse to either spatially separate a short fraction from the core pulse (as is the case with slicing), or to use time-of-flight dispersion to compress the entire pulse. So far electron bunch slicing at storage rings ${ }^{[8,13,14]}$ and electron bunch compression at free electron lasers (FEL) ${ }^{[15,16]}$ have been demonstrated.

A schematic view of the slicing setup at the SLS is shown in Fig. 1a. Femtosecond electron beam slicing is essentially a three-step process. ${ }^{[13]}$ First, an ultrashort laser pulse modulates the energy of a slice of relativistic electron bunch travelling through a periodic magnetic structure. In the second step, the bunch travels through energy dispersive bend magnets that separate the modulated electrons from the core beam. The energy modulated electrons then pass through a $19-\mathrm{mm}$-period in-vacuum undulator, emitting X-rays that are separated from those emitted by the core beam with appropriate apertures in the Xray beamline. $[17,18]$

The FEMTO laser system consists of a fs Ti:Sapphire oscillator synchronized to the SLS storage ring and two parallel regenerative amplifiers. The amplifiers deliver high energy fs pulses for both pumping samples and modulating the electron bunches inside the storage ring. In this way slicing provides inherently synchronized optical pump and X-ray probe pulses. A simulation of the $\sim 1 \%$ energy modulation imposed by the laser pulse on the electron bunch is shown in Fig. 1b. A diagnostic port installed at the first bending magnet after the interaction straight allows the extraction of coherent $\mathrm{THz}$ radiation emitted by the modulated electron bunches. ${ }^{[19]}$ The intensity of the $\mathrm{THz}$ radiation increases quadratically with the number of 


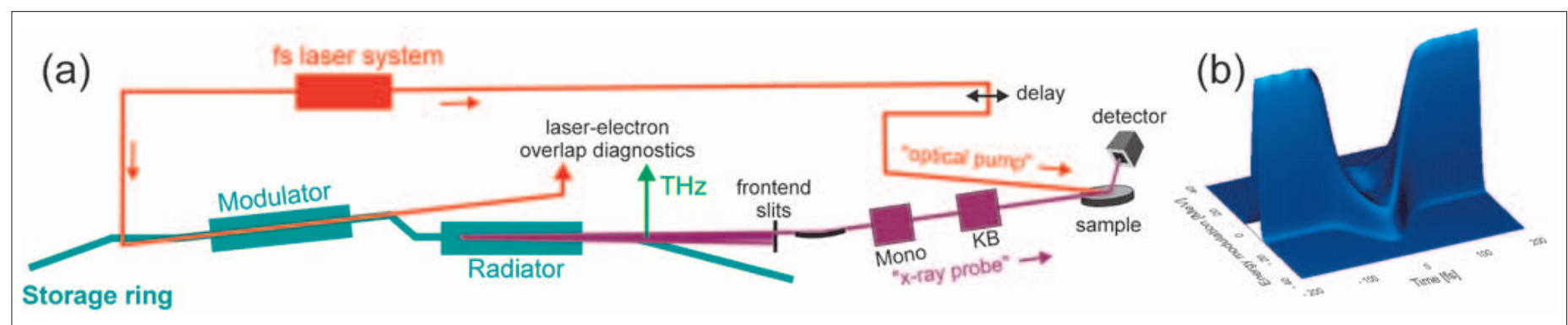

Fig. 1. (a) Schematic layout of the laser-X-ray pump-probe experiments at the microXAS beamline at the SLS. Mono: single crystal monochromator; KB: Kirkpatrick-Baez focusing system. (b) Simulation of the energy modulation on the $2.4 \mathrm{GeV}$ storage ring electrons in the storage ring using the $2 \mathrm{~mJ} 50 \mathrm{fs} 800 \mathrm{~nm}$ optical pulse provided by the laser system.

modulated electrons, providing a diagnostic tool to optimize and monitor the spatial and spectral laser-electron overlap. The fast transverse and longitudinal electron orbit feedback systems of the SLS storage ring, together with top-up mode operation, provide the stability needed to achieve micron-level position and micro-radian pointing stability of the X-ray beam. The stability of the source means that $200 \mathrm{fs}$ time resolution pump-probe experiments can be performed over many hours. This compensates somewhat for the low X-ray source flux, on the order of 5-10 $\times 10^{5}$ photons/s per $0.1 \%$ bandwidth.

\section{Femtosecond Grazing Incidence X-ray Diffraction}

In opaque solids, optical-wavelength photons are usually absorbed in a very thin surface layer ranging from 10-100 nanometers, whereas the attenuation length extends typically into the micrometer scale for X-ray wavelengths near $1 \AA$. One way to avoid this undesirable mismatch of excited and probed sample volume is to use thin films. ${ }^{[2]}$ In cases where the X-ray scattering is weak, the overall diffraction efficiency for thin films in a symmetric scattering geometry is very low. This is a serious disadvantage when using a low flux slicing source. In many cases a more efficient solution is to apply a grazingincidence, non-coplanar asymmetric diffraction geometry. ${ }^{[6]}$ This geometry allows one to match the absorption depth of the $\mathrm{X}$-ray probe to the laser excitation depth by setting the incidence angle of the X-rays to values near the critical angle for total external reflection.

\section{Coherent Phonon Dynamics}

Our initial experiments concentrated on laser induced coherent acoustic phonon motion such as strain waves in InSb and $\mathrm{Bi},{ }^{[20,21]}$ and on coherent optical phonons in $\mathrm{Bi}$ and Te. ${ }^{[6,8,21,22]}$ Large amplitude coherent optical phonons that can be generat- ed in bismuth with an ultrashort laser pulse via the displacive excitation mechanism have been studied by optical methods for many years. ${ }^{[22-25]}$ Recently this phenomenon has received renewed interest as a test ground for new ultrashort X-ray and electron pulse sources that can directly resolve the atomic motion using diffraction techniques. ${ }^{[2,6,8,26,27]}$ Progress has also been made in developing computational models. [28-30]

Bismuth, a semimetal with an indirect negative bandgap, has a two-atom rhombohedral unit cell that is slightly distorted from simple cubic symmetry due to the Jones-Peierls effect.[31] This distortion is very sensitive to electronic excitation, which tends to push the system towards higher structural symmetry. This motion of the atoms along the body diagonal of the rhombohedral unit cell corresponds to that of the symmetry preserving $A_{1 \mathrm{~g}}$ optical phonon mode. Excitation with a nearinfrared laser pulse significantly shorter than its phonon period of $\sim 300 \mathrm{fs}$ leads to a coherent, large amplitude $A_{1 \mathrm{~g}}$ mode. The frequency of this mode decreases significantly when increasing the optical pump intensity. ${ }^{[2,6,26]}$

One problem in understanding the excited-state dynamics of bismuth has been to disentangle the role of electronic transport dynamics (e.g. diffusion of carriers from the surface) from the relaxation of carrier energy via interaction with the lattice. The ability of femtosecond grazing incidence diffraction to probe the structural dynamics over different depth intervals can address this issue. Fig. 2 shows the observed intensity of the (111) Bragg reflection versus pump-probe delay for different incidence angles. Because electronic excitation causes the quasi-equilibrium position of the atoms to suddenly shift towards the higher symmetry structure, we observe strong, depth-dependant changes in the structure factor for diffraction from the (111) planes. The differences in the data as a function of incidence angle demonstrate the importance of control over the X-ray probe depth. By fitting the data using a model based on density functional theory ${ }^{[29]}$ we were able to extract an effective diffusion rate of $2.3 \pm 0.3 \mathrm{~cm}^{2} / \mathrm{s}$ for the excited carriers and an estimated electronhole interaction time of $260 \pm 20 \mathrm{fs}$. ${ }^{6]}$

XRD also allows mode-specific investigation of atomic motion. This is of particular interest in view of DFT calculations that predict at high excitation fluences a strong coupling between the $A_{1 \mathrm{~g}}$ and the degenerate $E_{\mathrm{g}}$ symmetry-breaking optical phonon. [29] Ôptical pump-probe experiments performed at $7 \mathrm{~K}$ have shown evidence of a coupling between the $A_{1 \mathrm{~g}}$ and $E_{\mathrm{g}}$ optical phonon modes. ${ }^{[25]}$ To investigate this and other types of structural dynamics in bismuth, we performed measurements of diffraction from the (1-10) and (1-21) lattice planes which are insensitive to the motion of the $A_{1 g}$ phonon coordinate. ${ }^{[32]}$ The data show evidence of an increase in lattice disorder with a time scale of less than $500 \mathrm{fs}$. This time scale is much faster than the time scale of lattice heating inferred from the behavior of the $A_{1 g}$ phonon coordinate, suggesting a non-thermal type of lattice disorder in this system. ${ }^{[6]}$

To investigate any possible coupling of this disorder with the coherent $A_{1 \mathrm{~g}}$ phonon, we applied a sequence of two pulses to control the population of the $A_{1 \mathrm{~g}}$ phonon mode. ${ }^{[32]}$ In this double-pump control experiment, the amplitude of the coherent atomic motion can be manipulated through the delay of the second pulse by keeping the electronic excitation constant. Any significant coupling from the $A_{1 \mathrm{~g}}$ mode to the disorder within the (111) plane should lead to a change of the corresponding Bragg diffraction signal when the pulse sequence is adjusted to either maximize or to cancel the amplitude of the coherent $A_{1 \mathrm{~g}}$ phonon.

The control of the $A_{1 \mathrm{~g}}$ amplitude for two different delay settings of the exciting pulse pair and the corresponding (1-21)-transients are shown in Fig. 3. Within the accuracy of our experiment we do not observe any change of the (1-21)-diffraction signal. We conclude that at room temperature coupling from the $A_{1 \mathrm{~g}}$ to the increase in lattice disorder within the (111) plane is not measurable. The observed fast decrease in diffraction efficiency ap- 

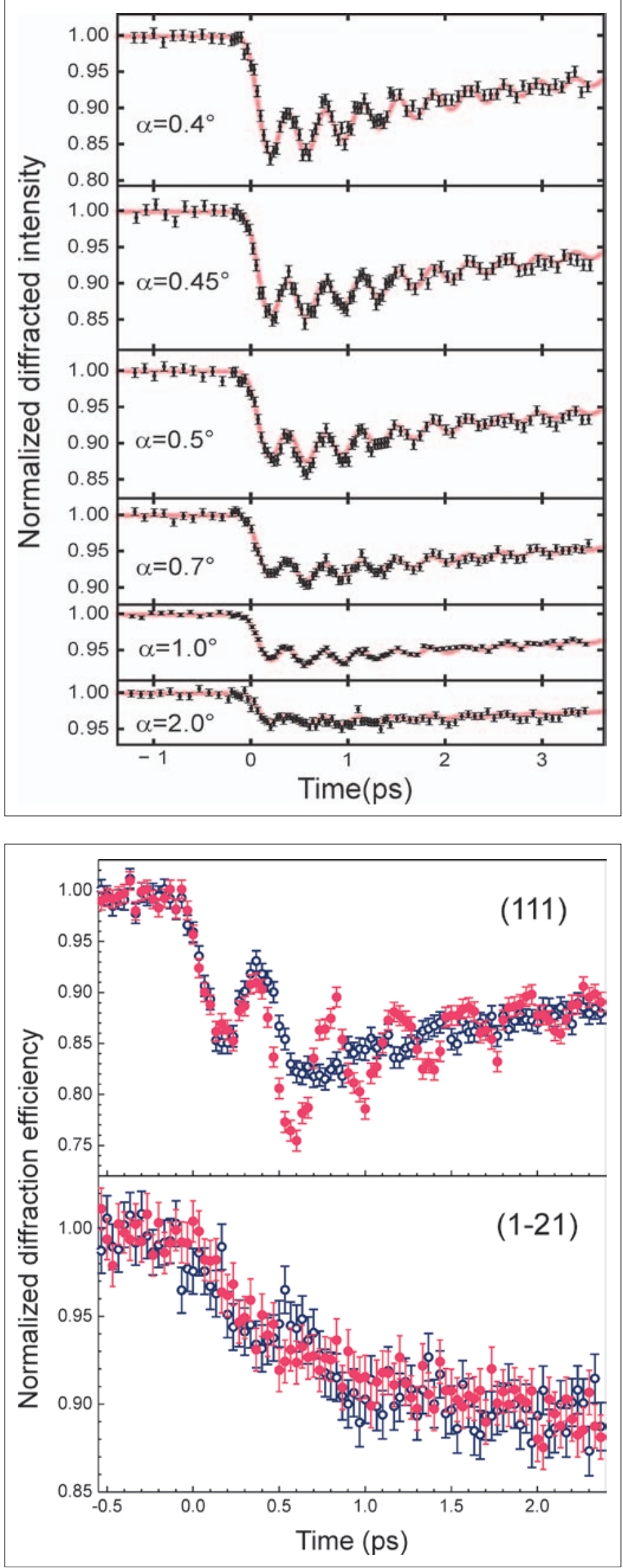

Fig. 3. Measured response of the $\mathrm{Bi}$ (111) and (1-21) Bragg peaks controlled by a two-pulse excitation scheme for a relative delay of 380 (full) and 575 fs (empty circles) between the pump pulse pair. The absorbed energy density was $1.1 \mathrm{~mJ} /$ $\mathrm{cm}^{2}$ for the first and $0.8 \mathrm{~mJ} / \mathrm{cm}^{2}$ for the second pulse, respectively. pears instead to be a direct consequence of electronic excitation of the crystal. This results in a nearly instantaneous change in the frequencies of vibrational modes throughout the Brillouin zone, causing a non-thermal increase of the rms displacements of random atomic motions within the (111) plane. Later experiments with more precision have revealed small oscillations in this non-equilibrium lattice noise, providing direct experimental evidence for a phenomenon known as 'phonon squeezing'. [33]

\section{Photo-induced Phase Transitions in Strongly Correlated Electron Systems}

In strongly correlated electron systems (SCES) the strong interplay between valence charge distribution, orbital order, magnetic order, and subtle distortions of the atomic lattice give rise to complex phase diagrams. The structural, electronic and/or magnetic long range order often is the result of competing atomic-scale interactions that lead to changes in crystal symmetry and to technologically relevant material properties such as high- $T$ superconductivity, colossal magnetoresistance and multiferroicity. Exciting the electronic system with a strong pulse of light can disturb this order and trigger non-thermal phase transitions on an ultrafast time scale. This opens new opportunities to investigate underlying correlations in the time domain and to control material properties on a subpicosecond time scale.

Recently, we have applied trXRD diffraction to observe direct and unambiguous structural motion due to laser induced charge- and orbital-order melting in a thin film $(d=55 \mathrm{~nm})$ of the three-dimensional manganite system $\mathrm{La}_{(1-x)} \mathrm{Ca}_{\mathrm{XnO}}$ (LCMO) $x \approx 0.5 .{ }^{[34]}$ For $x=0.58$, LCMO at room temperature is paramagnetic and undergoes upon cooling a phase transition to a charge and orbitally ordered phase $(\mathrm{T}<$ $\left.\mathrm{T}_{\mathrm{CO} / \mathrm{OO}} \approx 240 \mathrm{~K}\right) .^{[35]}$ The staggered structure of the O-octahedra at the $\mathrm{Mn}^{3+}$ sites shown in Fig. 4a is a result of a Jahn-Teller (J-T) distortion that induces a doubling of the unit cell along the $b$-axis as compared to the high-T orthorhombic Pbnm structure. This structural change leads, for $T<T_{\mathrm{CO} / \mathrm{OO}}$, to additional weak superlattice (SL) Bragg peaks. The time dependence of such a SL reflection is shown in Fig. 4b for two fluences. At low excitation fluences we observe the displacive excitation of a coherent optical $A_{\mathrm{g}}$ phonon mode that involves motion of the $\mathrm{La} / \mathrm{Ca}$ cations in the direction of the crystal $a$-axis. ${ }^{[37]}$ Under high excitation conditions we observe a complete phase transition occurring within $\sim 1$ ps as demonstrated by the disappearance of the $\mathrm{SL}$ reflection. The simultaneous increase of the non-SL Bragg peak intensity confirms this interpretation. The initial step of the phase transition, corresponding to an $80 \%$ drop of the SL reflection, occurs on a time scale of less than 200 fs (our experimental time resolution). This drop is attributed to motion of the $\mathrm{Mn}^{4+}$ octahedra triggered by the prompt release of the J-T distortion upon direct melting of 


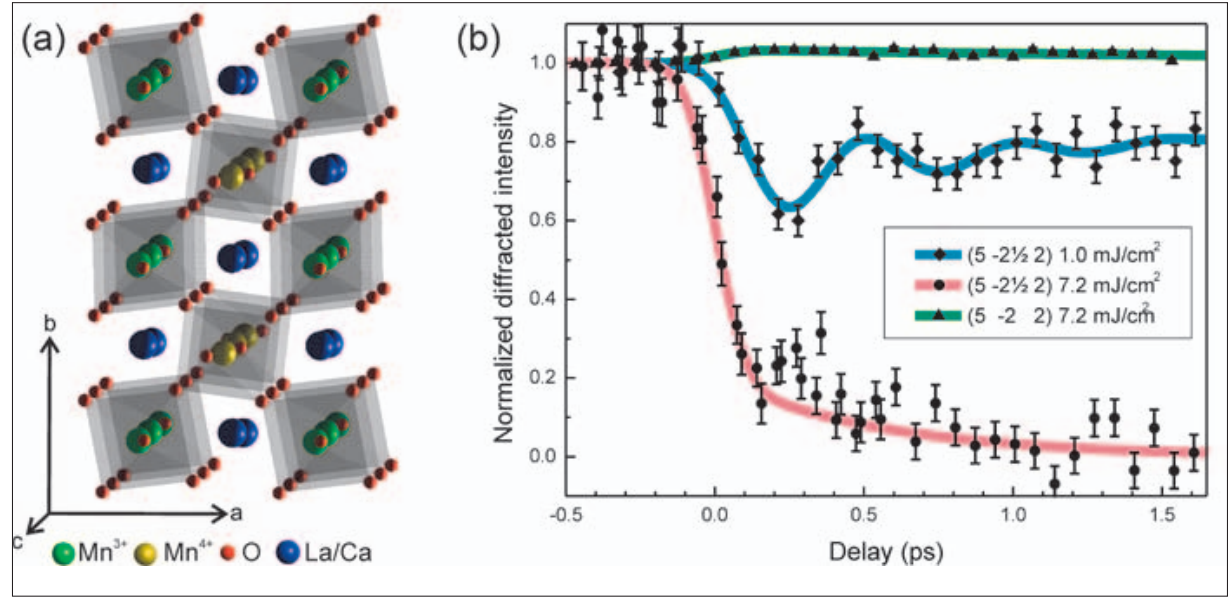

Fig. 4. (a) Ground state structure of $P 2_{1} \mathrm{~nm}$ symmetry of charge and orbitally ordered half-doped LCMO. $^{[36]}$ (b) Laser-induced response of the (5 -21/2 2) SL and (5 -2 2) non-SL Bragg reflections.

the charge and orbital order. The energy needed to launch this non-thermal phase transition is of the same order as that estimated to thermally melt the low- $T$ phase. This suggests that the J-T distortion is the main driving force for the rich manifold of electronic and magnetic order observed in these materials.

To understand these dynamics on a more detailed level, we intend to investigate different manganite systems over a wide parameter range. Complimentary to this, we plan to probe the response of the charge and orbital order with resonant soft $\mathrm{X}$-ray diffraction at existing $4^{\text {th }}$ generation soft X-ray sources that in the near future might offer a time resolution in the sub-10 fs range needed to fully resolve the underlying structural and electronic dynamics. The combination of structural and electronic information will help to separate correlated effects on an ultrafast time scale as they evolve to clarify the complex interplay between the electronic system and the lattice in these materials.

Charge density waves (CDW) comprise another class of collective phenomena arising from a correlation between the electron density and the underlying lattice. There exist several competing hypotheses for the mechanism driving the CDW formation in $1 T$-TiSe ${ }_{2}$, such as the band J-T effect, Fermi-surface nesting or exciton condensation. The latter becomes possible due to a low free carrier density and a consequently poorly screened Coulomb interaction. ${ }^{[38]}$ We studied the dynamics of the structural order parameter of the CDW in $\mathrm{TiSe}_{2}$ by time-resolved optical reflectivity and trXRD. ${ }^{[39]}$ In contrast to our experimental finding on the J-T driven manganite system discussed above we find that the energy needed to melt the CDW in $1 T$ $\mathrm{TiSe}_{2}$ is significantly lower than that expected for a thermal phase transition. This observation combined with the extremely fast time scale $(<200 \mathrm{fs})$ for the phase tran- sition, provides experimental support for the exciton condensation mechanism as the main driving force for the CDW formation in $1 T$-TiSe ${ }_{2}{ }^{[40]}$

Away from an absorption edge, X-rays interact predominantly with tightly bound core level electrons, providing information about the relative positions of atoms. The situation is somewhat different for X-ray energies close to an electronic transition. Resonant X-ray diffraction (RXRD) is a powerful technique to obtain direct information about charge, orbital and spin degrees of freedom. ${ }^{[41-43]}$ It combines the atomic resolution of XRD to probe longrange order with element specific sensitivity of XAS to probe local electronic configurations. Time resolved RXRD can study the dynamics of long-range order of magnetism, charge and orbitals. ${ }^{[12]}$ Using the high intensity of the X-ray pulses offered by FEL's time-resolved RXRD experiments may be pushed to the inelastic regime by applying resonant inelastic Xray scattering (RIXS) techniques to measure momentum transfer dispersion of collective magnetic excitations in long-range ordered magnetic systems. ${ }^{[44]}$

\section{THz-induced Dynamics}

So far we have used optical pulses with photon energies in the $1-3 \mathrm{eV}$ range to photo-excite samples, resulting in strong electronic excitations. The various relaxation mechanisms from this strong perturbation of the electronic states result in a sometimes complex chain of events which are often difficult to disentangle. Sources of short-pulse $\mathrm{THz}$ frequency radiation, now able to generate transient electric fields in the hundreds of $\mathrm{kV}$ range, ${ }^{[45]}$ opens up the possibility to perform two important types of experiments:

i) Resonant excitation of specific lattice modes with tunable $\mathrm{THz}$ light offers the opportunity to investigate the coupling from the lattice to the electronic system by resonantly exciting selected vibrational IR-active modes and by probing the lattice motion and corresponding changes of the electronic and magnetic order using time-resolved RXRD. ${ }^{[46]}$ This way no excess energy is dumped into the material as it is often required in optically pumped experiments. A metal-insulator transition in a manganite via mode selective-excitation has been demonstrated for $\operatorname{Pr}_{0.7} \mathrm{Ca}_{0.3} \mathrm{MnO}_{3} \cdot{ }^{.47]}$

ii) By taking advantage of the high electric fields offered by intense $\mathrm{THz}$ half cycle pulses it might even be possible to study the dynamical response of multiferroic materials to intense electric fields and even control their magnetic state without use of high electric currents. Multiferroics intrinsically exhibit a coupled ordering of a magnetization (arising from the electron spin and associated orbital magnetic moments) and of an electric polarization (arising from aspheric charge distribution). ${ }^{[48]}$ Using static resonant X-ray diffraction, such a coupling has directly been demonstrated in multiferroic $\mathrm{ErMn}_{2} \mathrm{O}_{5}$ by applying an electric field that pushes the system from its ferroelectric phase below TCM into the commensurate (CM) magnetic phase. ${ }^{[49]}$

\section{Summary and Outlook}

After five years of operation, the FEMTO source at the Swiss Light Source has made significant progress in developing trXRD methods. Current work focuses mostly on structural and magnetic dynamics in strongly correlated electron systems. The rapid technological development of new sources ${ }^{[50]}$ and techniques ${ }^{[51,52]}$ in the area of ultrafast structural research has and continues to stimulate tremendous growth of our ability to study the relationships between electronic, magnetic and structural order in correlated electron systems.

\section{Acknowledgements}

The authors acknowledge financial support from the Swiss NSF. The FEMTO slicing source was started by special funds provided by the ETH Board.

Received: March 7, 2011

[1] A. Cavalleri, C. Tóth, C. W. Siders, J. A. Squier, F. Ráksi, P. Forget, J. C. Kieffer, Phys. Rev. Lett. 2001, 87, 237401.

[2] K. Sokolowski-Tinten, C. Blome, J. Blums, A. Cavalleri, C. Dietrich, A. Tarasevitch, I Uschmann, E. Förster, M. Kammler, M. Hornvon-Hoegen, D. von der Linde, Nature, 2003, 422, 287.

[3] A. Cavalleri, M. Rini, H. H. W. Chong, S Fourmaux, T. E. Glover, P. A. Heimann, J. C. 
Kieffer, R. W. Schoenlein, Phys. Rev. Lett. 2005, 95, 067405.

[4] C. v. Korff-Schmising, M. Bargheer, M. Kiel, N. Zhavoronkov, M. Woerner, T. Elsaesser, I. Vrejoiu, D. Hesse, M. Alexe, Phys. Rev. Lett. 2007, 98, 257601.

[5] C. Stamm, T. Kachel, N. Pontius, R. Mitzner, T. Quast, K. Holldack, S. Khan, C. Lupulescu, E. F. Aziz, M. Wietstruk, H. A. Durr, W. Eberhardt, Nature Mater. 2007, 6, 740.

[6] S. L. Johnson, P. Beaud, C. J. Milne, F. S. Krasniqi, E. S. Zijlstra, M. E. Garcia, M. Kaiser, R. Abela, G. Ingold, Phys. Rev. Lett. 2008, 100 155501.

[7] T. Elsaesser, M. Woerner, Acta Crystallogr. A 2010, 66, 168.

[8] P. Beaud, S. L. Johnson, A. Streun, R. Abela, D. Abramsohn, D. Grolimund, F. Krasniqi, T. Schmidt, V. Schlott, G. Ingold, Phys. Rev. Lett. 2007, 99, 174801.

[9] C. Bressler, C. Milne, V. T. Pham, A. ElNahhas, R. M. van der Veen, W. Gawelda, S. L. Johnson, P. Beaud, D. Grolimund, C. Borca, G. Ingold, R. Abela, M. Chergui, Science 2009, 323, 489.

[10] M. Chergui, Acta Crystallogr. A 2010, 66, 229.

[11] E. Vorobeva, S. L. Johnson, P. Beaud, C. J. Milne, M. Benfatto, G. Ingold, Phys Rev. B 2009, 80, 134301.

[12] G. Ingold, R. Abela, P. Beaud, S. L. Johnson, U. Staub, Z. Kristallogr. 2008, 223, 292.

[13] R. W. Schoenlein, S. Chattopadhyay, H. H. W. Chong, T. E. Glover, P. A. Heimann, C. V. Shank1, A. A. Zholents, M. S. Zolotorev, Science 2000, 287, 2237.

[14] S. Khan, K. Holldack, T. Kachel, R. Mitzner, T. Quast, Phys. Rev. Lett. 2006, 97, 074801.

[15] W. Ackermann, G. Asova, V. Ayvazyan, A. Azima, N. Baboi, J. Bähr, V. Balandin, B. Beutner, A. Brandt, A. Bolzmann, R. Brinkmann, O. I. Brovko, M. Castellano, P. Castro, L. Catani, E. Chiadroni, S. Choroba, A. Cianchi, J. T. Costello, D. Cubaynes, J. Dardis, W. Decking, H. Delsim-Hashemi, A. Delserieys, G. Di Pirro, M. Dohlus, S. Düsterer, A. Eckhardt, H. T. Edwards, B. Faatz, J. Feldhaus, K. Flöttmann, J. Frisch, L. Fröhlich, T. Garvey, U. Gensch, Ch. Gerth, M. Görler, N. Golubeva, H.-J. Grabosch, M. Grecki, O Grimm, K. Hacker, U. Hahn, J. H. Han, K. Honkavaara, T. Hott, M. Hüning, Y. Ivanisenko, E. Jaeschke, W. Jalmuzna, T. Jezynski, R. Kammering, V. Katalev, K. Kavanagh, E. T. Kennedy, S. Khodyachykh, K. Klose, V. Kocharyan, M. Körfer, M. Kollewe, W. Koprek, S. Korepanov, D. Kostin, M. Krassilnikov, G. Kube, M. Kuhlmann, C. L. S. Lewis, L. Lilje, T. Limberg, D. Lipka, F. Löhl, H. Luna, M. Luong, M. Martins, M. Meyer, P. Michelato, V. Miltchev, W. D. Möller, L. Monaco, W. F. O. Müller, O. Napieralski, O. Napoly, P. Nicolosi, D. Nölle, T. Nuñez, A. Oppelt, C. Pagani, R. Paparella, N. Pchalek, J. Pedregosa-Gutierrez, B. Petersen, B. Petrosyan, G. Petrosyan, L. Petrosyan, J. Pflüger, E. Plönjes, L. Poletto, K. Pozniak, E. Prat, D. Proch, P. Pucyk, P. Radcliffe, H. Redlin, K. Rehlich, M. Richter, M. Roehrs, J. Roensch, R. Romaniuk, M. Ross, J. Rossbach, V. Rybnikov, M. Sachwitz, E. L. Saldin, W. Sandner, H. Schlarb, B. Schmidt, M. Schmitz, P. Schmüser, J. R. Schneider, E. A. Schneidmiller, S. Schnepp, S. Schreiber, M. Seidel, D. Sertore, A. V. Shabunov, C. Simon, S. Simrock, E. Sombrowski, A. A. Sorokin, P. Spanknebel, R. Spesyvtsev, L. Staykov, B. Steffen, F. Stephan, F. Stulle, H. Thom, K. Tiedtke, M. Tischer, S. Toleikis, R. Treusch, D. Trines, I. Tsakov, E. Vogel, T. Weiland, H. Weise, M. Wellhöfer, M. Wendt, I. Will, A. Winter, K. Wittenburg, W. Wurth, P. Yeates, M. V. Yurkov, I. Zagorodnov, K. Zapfe, Nature Photonics 2007, 1, 336.
[16] P. Emma, R. Akre, J. Arthur, R. Bionta, C. Bostedt, J. Bozek, A. Brachmann, P. Bucksbaum, R. Coffee, F.-J. Decker, Y. Ding, D. Dowell, S. Edstrom, A. Fisher, J. Frisch, S. Gilevich, J. Hastings, G. Hays, P. Hering, Z Huang, R. Iverson, H. Loos, M. Messerschmidt, A. Miahnahri, S. Moeller, H.-D. Nuhn, G. Pile, D. Ratner, J. Rzepiela, D. Schultz, T. Smith, P. Stefan, H. Tompkins, J. Turner, J. Welch, W. White, J. Wu, G. Yocky, J. Galayda, Nature Photonics 2010, 4, 641.

[17] G. Ingold, P. Beaud, S. Johnson, A. Streun, R. Abela, A. AlAdwan, D. Abrahamson, M. Boege, D. Grolimund, A. Keller, L. Rivkin, T. Schilcher, T. Schmidt, V. Schlott, L. Schulz, F. Van der Veen, D. Zimoch, AIP Conf. Proc. 2007, 879, 1198.

[18] G. Ingold, P. Beaud, S. L. Johnson, D. Grolimund, V. Schlott, T. Schmidt, A. Streun, Synchr. Rad. News 2007, 20, 39.

[19] K. Holldack, S. Khan, R. Mitzner, T. Quast, Phys. Rev. Lett. 2006, 96, 054801.

[20] F. S. Krasniqi, S. L. Johnson, P. Beaud, M. Kaiser, D. Grolimund, G. Ingold, Phys. Rev. B 2008, 78, 174302 .

[21] S. L. Johnson, P. Beaud, E. Vorobeva, C. J. Milne, E. D. Murray, S. Fahy, G. Ingold, Acta Crystallogr. A 2010, 66, 157.

[22] S. L. Johnson, E. Vorobeva, P. Beaud, C. J. Milne, G. Ingold, Phys. Rev. Lett. 2009, 103, 205501.

[23] H. J. Zeiger, J. Vidal, T. K. Cheng, E. P. Ippen, G. Dresselhaus, M. S. Dresslhaus, Phys. Rev. B 1992, 45, 768 .

[24] M. Hase, M. Kitajima, S. Nakashima, K. Mizoguchi, Phys. Rev. Lett. 2002, 88, 067401.

[25] O. V. Misochko, K. Ishioka, M. Hase, M. Kitajima, J. Phys.: Condens. Matter 2006, 18, 10571.

[26] D. M. Fritz, D. A. Reis, B. Adams, R. A. Akre, J. Arthur, C. Blome, P. H. Bucksbaum, A. L. Cavalieri, S. Engemann, S. Fahy, R. W. Falcone, P. H. Fuoss, K. J. Gaffney, M. J. George, J. Hajdu, M. P. Hertlein, B. Hillyard, Horn-von Hoegen, M. Kammler, J. Kaspar, R. Kienberger, P. Krejcik, S. H. Lee, A. M. Lindenberg, B. McFarland, D. Meyer, T. Montagne, É. D. Murray, A. J. Nelson, M. Nicoul, R. Pahl, J. Rudati, H Schlarb, D. P. Siddons, K. Sokolowski-Tinten, T. Tschentscher, D. von der Linde, J. B. Hastings, Science 2007, 315, 633.

[27] G. Sciaini, M. Harb, Se. G. Kruglik, T. Payer, C. T. Hebeisen, F.-J. Meyer zu Heringdorf, M. Yamaguchi, M. Horn von Hoegen, R. Ernstorfer, R. J. D. Miller, Nature 2009, 458, 56

[28] S. Fahy, D. A. Reis, Phys. Rev. Lett. 2004, 93, 109701

[29] E. S. Zijlstra, L. L. Tatarinova, M. E. Garcia, Phys. Rev. B 2006, 74, 220301

[30] É. D. Murray, S. Fahy, D. Prendergast, T. Ogitsu, D. M. Fritz, D. A. Reis, Phys. Rev. B 2007, 75, 184301 .

[31] R. E. Peierls, 'More Surprises in Theoretical Physics', Princeton University Press, 1991.

[32] P. Beaud, S. L. Johnson, C. J. Milne, F. Krasniqi, E. Vorobeva, G. Ingold, Springer Ser. Chem. Phys. 2009, 92, 104.

[33] S. L. Johnson, P. Beaud, E. Vorobeva, C. J. Milne, E. D. Murray, S. Fahy, G. Ingold, Phys. Rev. Lett. 2009, 102, 175503.

[34] P. Beaud, S. L. Johnson, E. Vorobeva, U. Staub, R. A. De Souza, C. J. Milne, Q. X. Jia, G. Ingold, Phys. Rev. Lett. 2009, 103, 155702.

[35] S.-W. Cheong, H. Y. Hwang, in 'Colossal Magnetoresistance Oxides', Ed. Y. Tokura, Gordon and Breach, London 1999, Chap. 7.

[36] E. E. Rodriguez, T. Proffen, A. Llobet, J. J. Rhyne, J. F. Mitchell, Phys. Rev. B 2005, 71, 104430.

[37] V. A. Amelitchev, B. Güttler, O. Yu. Gorbenko, A. R. Kaul, A. A. Bosak, A. Yu. Ganin, Phys. Rev. B 2001, 63, 104430.
[38] H. Cercellier, C. Monney, F. Clerc, C. Battaglia, L. Despont, M. G. Garnier, H. Beck, P. Aebi, L. Patthey, H. Berger, L. Forró, Phys. Rev. Lett. 2007, 99, 146403.

[39] E. Vorobeva, S. L. Johnson, P. Beaud, U. Staub, R. De Souza, C. Milne, J. Demsar, H. Schäfer, A. Titov, G. Ingold, in 'Ultrafast Phenomena XVII', Eds. M. Chergui, D. Jonas, E. Riedle, R. Schoenlein, A. Taylor, Oxford University Press 2011, 164.

[40] E. Vorobeva, S. L. Johnson, P. Beaud, U. Staub, R. De Souza, C. J. Milne, G. Ingold, J. Demsar, H. Schaefer, A. Titov, 2011, submitted.

[41] U. Staub, G. I. Meijer, F. Fauth, R. Allenspach, G. Bednorz, J. Karpinski, S. M. Kazakov, L. Paolasini, F. d'Acapito, Phys. Rev. Lett. 2003 $88,126402$.

[42] S. Grenier, K. J. Thomas, J. P. Hill, U. Staub, Y Bodenthin, M. García-Fernández, V. Scagnoli, V. Kiryukhin, S.-W. Cheong, B. G. Kim, J. M. Tonnerre, Phys. Rev. Lett. 2007, 99, 206403.

[43] U. Staub, V. Scagnoli, Y. Bodenthin, M. GarcíaFernández, R. Wetter, A. M. Mulders, H. Grimmer, M. Horisberger, J. Syn. Rad. 2008 $15,469$.

[44] J. Schlappa, T. Schmitt, F. Vernay, V. N Strocov, V. Ilakovac, B. Thielmann, H. M. Ronnow, S. Vanishri, A. Pazzalunga, X. Wang, L. Braicovich, G. Ghiringhelli, C. Marin, J. Mesot, B. Delley, L. Patthey, Phys. Rev. Lett. 2009, 103, 047401

[45] T. Bartel, P. Gaal, K. Reimann, M. Woerner, T. Elsaesser, Opt. Lett. 2005, 30, 2805.

[46] A. Cavalleri, S. Wall, C. Simpson, E. Statz, D. W. Ward, K. A. Nelson, M. Rini, R. W Schoenlein, Nature 2006, 442, 664.

[47] M. Rini, R. Tobey, N. Dean, J. Itatani, Y Tomioka, Y. Tokura, R. W. Schoenlein, A. Cavalleri, Nature 2007, 449, 72.

[48] S.-W. Cheong, M. Mostovoy, Nature Mater. 2007, 6, 13.

[49] Y. Bodenthin, U. Staub, M. Garcia-Fernandez, M. Janoschek, J. Schlappa, E. I. Golovenchits, V. A. Sanina, S. G. Lushnikov, Phys Rev. Lett. 2008, 100, 027201.

[50] http://www.psi.ch/swissfel

[51] M. Eichberger, H. Schäfer, M. Krumova, M. Beyer, J. Demsar, H. Berger, G. Moriena, G. Sciaini, R. J. D. Miller, Nature 2010, 468, 799.

[52] J. C. Petersen, S. Kaiser, N. Dean, A. Simoncig, H. Liu, A. L. Cavalieri, C. Cacho, I. C. E. Turcu, E. Springate, F. Frassetto, L. Poletto, S. S. Dhesi, H. Berger, A. Cavalleri, arXiv:1010.5027v1, 2010. 